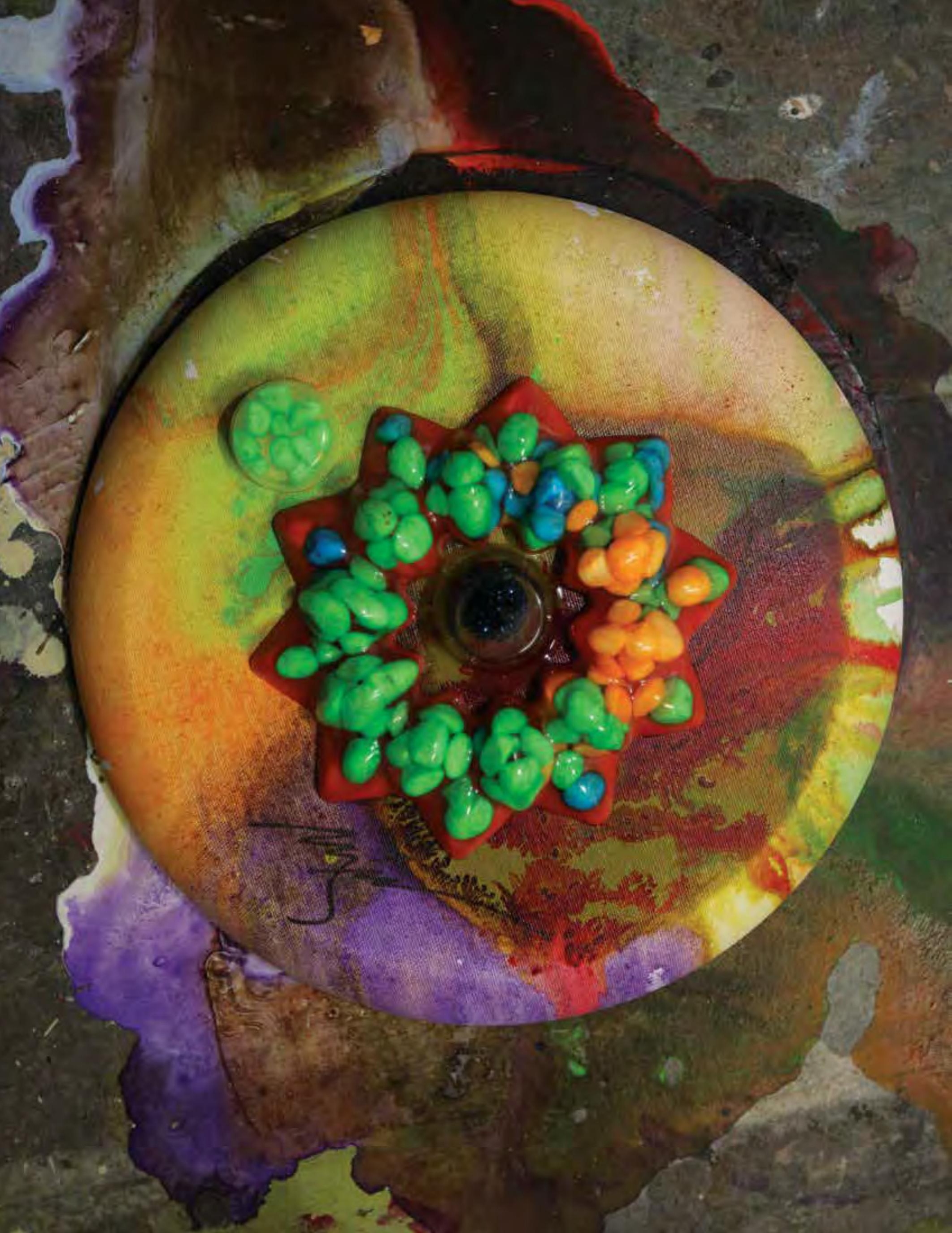


"Cuando haya muerto, llórame tan solo......"

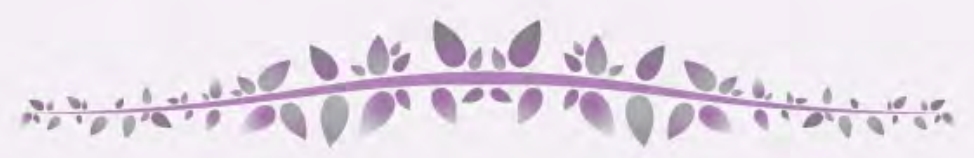

Cuando haya muerto, llórame tan sólo mientras escuches la campana triste, anunciadora al mundo de mi fuga del mundo vil hacia el gusano infame.

Y no evoques, si lees esta rima, la mano que la escribe, pues te quiero tanto que hasta tu olvido prefiriera a saber que te amarga mi memoria.

Pero si acaso míras estos versos cuando del Garro nada me separe, ni síquiera mi pobre nombre digas y que tu amor conmigo se marchite, para que el sabio en tu llorar no indague y se burle de ti por el ausente.

William Shakespeare Dramaturgo, poeta y actor inglés 


\title{
Años de Vida Potencialmente Perdidos por Cáncer de Mama. Hospital San Felipe de Tegucigalpa, Honduras, 2015
}

\author{
MARÍA GUADALUPE ROMERO-A¹, / INGRID CAROLINA GARAY-R². \\ Recibido 03.05.16 / Aprobado 08.07.16
}

\begin{abstract}
RESUMEN. El cáncer de mama en mujeres es una de las causas más importantes de mortalidad en el mundo y de igual forma en Honduras. Los países han procurado promover aspectos de índole preventivo entre la población, pero no se ha logrado evidenciar el impacto de estas acciones. La medición de la mortalidad se realiza con diferentes técnicas y el fenómeno de la mortalidad, representa un indicador sensible que evidencia las características generales de un grupo de pacientes, especialmente en lo relacionado a los servicios de salud. En este trabajo se tiene como objetivo dar a conocer el impacto de la mortalidad por cáncer de mama en el Hospital San Felipe de la ciudad de Tegucigalpa durante el período de 2015. Para ello, se emplea el cálculo de Años de Vida Potencialmente Perdidos (AVPP) con el fin de establecer la prematurez de muerte por esta causa específica. Es un estudio descriptivo, transversal y retrospectivo. Los datos fueron tomados de los expedientes clínicos a partir de los cuales, se agrupa la edad, se establece la procedencia geográfica en forma departamental y municipal y se evidencian factores de riesgo, como ser: adicciones al tabaco y al alcohol, menarquia antes de los once años, presencia de menopausia al momento del ingreso, condiciones socioeconómicas, utilización de servicios de salud. Los resultados muestran que el cáncer de mama desde el punto de la tasa neta de mortalidad hospitalaria, ocupa el segundo lugar en la casuística de ese año. El cálculo de AVPP es de 379 años.
\end{abstract}

Palabras Clave: cáncer de mama, mortalidad neta hospitalaria, años de vida potencialmente perdidos.

ABSTRACT. Breast cancer in women is one of the most important causes of mortality in the world and similarly in Honduras. Countries have sought to promote preventive aspects of nature among the population, but has not been able to demonstrate the impact of these actions. Measuring mortality is performed with different techniques and the phenomenon of death, is a sensitive indicator that shows the general characteristics of a group of patients, especially in relation to health services. This paper aims to raise awareness of the impact of mortality from breast cancer in the San Felipe Hospital in Tegucigalpa in 2015. For this period, the calculation of disability-adjusted life year is used (AVPP) in order to establish death prematurity this specific cause. A descriptive, cross-sectional and retrospective study. The data are taken from the clinical records from which the age groups, geographical origin is established as departmental and municipal and risk factors are evident, such as: addictions to snuff and alcohol, menarche before eleven, presence of menopause at admission, socioeconomic conditions, use of health services. The results show that breast cancer from the point of the net rate of hospital mortality, ranks second in the casuistry of that year. LPYL is 379 years.

Keywords: breast cancer, net hospital mortality, disability- adjusted life year.

\section{Introducción}

Los análisis de los niveles y tendencias de la mortalidad son instrumentos básicos para la planificación y el establecimiento de políticas gubernamentales en los diferentes campos, a su vez, muestra la heterogeneidad de condiciones de vida entre las distintas poblaciones (Agudelo, Aguirre, \& Dávila, 2010).

El estudio de la mortalidad, evidencia un indicador sensible que pone de manifiesto las características generales de los individuos específicamente en el ámbito socioeconómico y sanitario.
Hasta el 5\% de la carga de la enfermedad se relaciona con los tumores malignos en el mundo. En los países ricos, esta cifra se eleva al $14.6 \%$ y disminuye a $2.2 \%$ en los pobres. La diferente causa atribuible a los tumores malignos entre los países ricos y los países pobres es notoria, de tal forma que, en los países ricos, el riesgo de muerte es de 2.4 veces más que en los países pobres y de ingresos medios, reflejando con ello, una cantidad en años de vida potencialmente perdidos por muerte prematura por esta causa (Lozano-Asencio Rafael, 2008).

Cuando la causa de muerte es el cáncer de mama, el $1 \%$ de la carga de la enfermedad en las mujeres, se vincula

Médico y Cirujano. Máster en Salud Pública. Máster en Administración de Servicios de Salud. PhD. mgromeroa@yahoo.com

. Ingeniera en Ciencias Ambientales. Máster en Ciencias, Tecnología y Gestión del Agua. PhD. icgaray@usal.es 
con esta causa a nivel mundial y representa el $3.2 \%$ en los países ricos y el $0.4 \%$ en los pobres (Lozano-Asencio Rafael, 2008).

El incremento del riesgo de padecer y morir por esta causa en países pobres y de ingresos medios, se vincula con cambios en los patrones reproductivos y nutricionales sumados a la disminución de la fecundidad, los cambios en los estilos de vida y las condiciones de trabajo en las mujeres (Lozano-Asencio Rafael, 2008).

El cáncer de mama, bajo estas condiciones, se convierte en un reto emergente y en un horizonte de mucho trabajo para la salud de las mujeres de los países en vías de desarrollo. Generalmente, los problemas de salud del mundo en estos países se focalizan en los pobres, pero el cáncer de mama afecta a las mujeres adultas de todos los niveles económicos y en las dos etapas de pre y post menopausia de la vida (Knaul, Bustreo, Ha, \& Langer, 2009). La carga de salud para las mujeres pobres es doble, ya que hay una alta concentración de mortalidad materna persistente, altas prevalencias de VIH/SIDA y de cáncer de cuello uterino entre las mujeres de edad reproductiva.

La magnitud de la amenaza del cáncer de mama en la mujer es en gran parte desconocida por la falta de un adecuado registro de cáncer en aquellas mujeres que tienen la oportunidad de acceder a los servicios de salud o por deficiencias en programas de prevención que permiten una detección temprana utilizando tecnología avanzada tal como la mamografía, la que puede resultar sumamente costosa cuando el cáncer se encuentra en estadios avanzados.

Datos de Honduras, proporcionados por la Organización Mundial de la Salud (OMS), para el año 2012 y publicados en el 2014, reflejan que la relación de muerte por cáncer entre hombres y mujeres, es de 1:1. El cáncer de mama, ocupa el quinto lugar como causa de muerte por cáncer en el país, con un promedio de $8.2 \%$ del total, ocupando el primer lugar, el denominado otros cánceres, con un porcentaje de 46.5\% (OMS, Perfiles oncológicos de los países, 2014).

El propósito de este artículo es llamar la atención acerca del abordaje que se realiza a través de la detección de factores de riesgo y el impacto que representa para el sistema de salud la mortalidad por cáncer de mama en el Hospital San Felipe; el centro de referencia nacional para cáncer en el sistema de salud de Honduras. Para ello, se emplea el cálculo de Años de Vida Potencialmente Perdidos (AVPP).

El AVPP es un indicador con el cual se trata de determinar cuántos años de vida en promedio pierde una población en forma prematura por la muerte de las personas a determinadas edades y por diferentes causas utilizando como referente la esperanza de vida de la población para ese año y para el país. Esta técnica ha sido poco empleada en Honduras, sobre todo, en los hospitales (Agudelo, Aguirre, \& Dávila, 2010) (BID, OPS, \& Mundial, 2001).

\section{Metodología}

Este en un estudio descriptivo, transversal y retrospectivo sobre el comportamiento de los factores de riesgo y la mortalidad por cáncer de mama en las mujeres que ingresan a las salas de hospitalización del Hospital San Felipe; centro nacional de referencia de cáncer en el país. Se presentan sólo datos de los casos de mujeres, aunque este tipo de cáncer también puede afectar a los hombres.

Los datos tomados forman parte del sistema de información hospitalario para el año 2015. El proceso de recopilación se realizó a través de la revisión de los expedientes de los pacientes que fallecieron en el período indicado. Los datos que contribuyen a la investigación fueron tomados de la revisión de la historia clínica de ingreso, notas de evolución, exámenes de laboratorio, reporte de exámenes especiales, diagnósticos de la hoja de egreso, revisión del certificado de defunción y de la epicrisis (información que forma parte del resumen de la historia clínica del paciente que ha recibido servicios de emergencia o de hospitalización).

Entre los factores de riesgo se incluyen, el sexo, la edad y procedencia, el estado civil y ocupación, motivo de la consulta, presencia de menopausia, antecedentes personales de cáncer de mama, antecedentes familiares de cáncer de mama, menarquia antes de los 11 años, consumo de anticonceptivos orales, consumo de alcohol, paridad, tabaquismo y datos relacionados con el acceso a los servicios de salud, v.g., realización de mamografía, biopsia, quimioterapia, mastectomía y radioterapia. Con los datos anteriores se construyeron tablas de frecuencia por grupos de edad a partir de los 25 años hasta los 80 años.

Para el cálculo de la mortalidad neta se utilizó la fórmula entre el número de fallecidos en el servicio de más de 48 horas sobre el total de egresos en el servicio o en el Hospital por 100. Para el cálculo de la mortalidad bruta se utilizó la fórmula de número de fallecidos en el servicio o en el hospital sobre el total de egresos en el servicio o en el hospital (SESAL, 2010).

Para el cálculo de la Letalidad, se utilizó la fórmula de fallecidas por cáncer sobre ingresados por cáncer (SESAL, 2010). 
Para el cálculo de los AVPP, la fórmula que se utilizó fue:

$$
\begin{aligned}
& \mathbf{L} \\
& \mathbf{A V P P}=\sum(\mathrm{L}-\mathrm{Xi})
\end{aligned}
$$

Donde $\boldsymbol{L}=$ es el límite potencial de la vida (71.1 para hombres y 78.3 para mujeres).

(SESAL, Anuario Estadístico Año 2014, 2014).

$\boldsymbol{X}=$ es la edad de defunción del sujeto.

La esperanza de vida al nacer como valor límite de vida se aplicó para el cálculo de los AVPP de acuerdo al dato proporcionado por la Secretaría de Salud para el año 2014, en el Anuario Estadístico que publican. La limitante que presenta esta aproximación al cálculo de los AVPP es la no comparabilidad con otras poblaciones que seguramente tienen diferentes esperanzas de vida. No se pueden comparar los APVP entre dos o más situaciones a estudiar si el criterio de cálculo no es el mismo (OPS, 2003).

Para esta investigación, se consideró como límite de edad, los 25 años y los 79 años de edad (límite máximo). Se parte del supuesto de que las pacientes que murieron deberían haber vivido hasta la edad superior del intervalo de edades dentro del que se analiza la mortalidad (Agudelo, Aguirre, \& Dávila, 2010), razón por la cual, no se incluyen en el cálculo a las pacientes que fallecieron en edades superiores a la esperanza de vida del país para el año que está en observación. La esperanza de vida que se utiliza como límite superior en la fórmula es la que proporciona la Secretaría de Salud e informa como dato oficial para el año 2014, siendo esta de 74.6 para la población en general. Para hombres es de 71.1 y para mujeres, de 78.3 por lo que se considerará 79 años como edad límite (SESAL, 2014). Se tomaron datos de cáncer de mama en mujeres, ya que el $100 \%$ de las muertes del año que se observa, es de mujeres.

Los AVPP se pueden analizar de diferentes formas: mirando el valor del AVPP en cada grupo de edad o evaluando el total para la población o bien estudiando el valor del AVPP para una causa específica. El uso de AVPP se obtiene sumando el producto del número de muertes de cada edad por la diferencia entre esta edad y una edad límite. Esta sumatoria se expresa en AVPP.

Para los grupos de edad se asume que las muertes ocurren de manera uniforme en el periodo de edad, por lo que puede haber algunas diferencias entre los cálculos a partir de individuos o de grupos de individuos. Lo habitual es hacerlo por datos agrupados. Lo más común es considerar que las muertes ocurren en la edad correspondiente al punto medio de los grupos de edad (Toro Zapata, García Areiza, Sanchez Mora, \& Moren Florian, 2005).

\section{Aspectos conceptuales}

Algunos conceptos que se han utilizado en el desarrollo de la investigación son los siguientes:

\section{Defunción}

Es el fallecimiento de toda persona viva independiente de todas las horas que haya vivido.

\section{Tasa de letalidad}

La letalidad cuantifica la gravedad de una enfermedad. Indica el número de defunciones que acontecen por una causa determinada. El cálculo a nivel de atención hospitalario, implica la utilización como denominador del número total de egresos o el número de egresos por causa y se define como el porcentaje de casos de una enfermedad o de un evento determinado que mueren en un período específico (Gran \& Torres, 2010).

Tasa de Letalidad:

Fallecidas por cáncer de mama/egresados/por cáncer x 100$\mathrm{TL}=14 / 1,746 \mathrm{X} 100$ egresos $=0.801$

\section{Mortalidad Hospitalaria Bruta y Neta}

Expresa la calidad de la atención médica a nivel hospitalario. La mortalidad bruta o total expresa el riesgo de morir con respecto al total de egresos acontecidos, la mortalidad neta tiene idéntico propósito, pero circunscribiendo este riesgo a los ingresados de 48 horas o más. El cálculo se realiza a través del cociente del número de defunciones hospitalarias totales (mortalidad bruta) de 48 horas y más de hospitalización (tasa neta) y el número total de egresos para un hospital y período dado multiplicado por 100. El indicador se desagrega para las diferentes salas y servicios. El tipo de indicador es tasa (SESAL, 2010).

\section{Mortalidad prematura}

Años de vida potencialmente perdidos.

Son los años que una persona deja de vivir si fallece a una edad que no es la habitual de defunción fijada numéricamente para ese colectivo. Es una medida de la mortalidad que teóricamente se podría evitar. Los años de vida potencialmente perdidos es una forma de medición de la carga de una enfermedad, conceptualizándose así a la medida de pérdidas de salud que para una población representan las consecuencias mortales y no mortales de las diferentes enfermedades y lesiones. La carga de la enfermedad atribuible a una enfermedad concreta se mide por un lado con su frecuencia y por otro lado, a partir de las consecuencias mortales y discapacitantes que origine.

Uno de los indicadores más utilizados para valorar las muertes tempranas son los Años Potenciales de Vidas Perdidos (AVPP). El concepto de AVPP fue utilizado por primera vez por Dempsey (M., 1947) en 1947 para medir 
los cambios en la mortalidad producida por tuberculosis y compararla con las producidas por enfermedades cardíacas y cáncer. En 1950, Haenszel (W., 1950) observó que las tasas de muerte convencionales no proporcionaban suficiente peso a la mortalidad en edades jóvenes y propuso un índice de mortalidad que expresara las muertes en años de vida perdidos (Del Valle Gómez María Olivo, 1993).

Los años de vida potencialmente perdidos es una de las medidas del impacto relativo de varias enfermedades y problemas de salud en la sociedad que ilustran sobre las pérdidas que sufre la sociedad como consecuencia de la muerte de personas jóvenes o de fallecimientos prematuros. Se considera que una muerte es prematura cuando ocurre antes de cierta edad predeterminada que corresponde, por ejemplo, a la esperanza de vida al nacer en una población estudiada. Considerar la edad a la cual mueren las personas y no solo el evento mismo de la muerte permite asignar un peso diferente a las muertes que ocurren a diferentes momentos de la vida (Velásquez, 2006).

\section{Discución de resultados}

4.1 Aspectos sociodemográficos de las pacientes que murieron por cáncer de mama en el Hospital San Felipe

\section{- Procedencia de los pacientes que fallecieron por cáncer} de mama en el HSF

El porcentaje más importante de mortalidad por cáncer de mama corresponde al departamento de Francisco Morazán con un 50\%, lo que representa la mitad de los datos observados; en el segundo lugar, se encuentran los departamentos de Cortés y Choluteca con el $14.3 \%$ respectivamente y, en el tercer lugar, se encuentran los departamentos de Comayagua y Olancho con el 7.1\% respectivamente (Cuadro $\mathrm{N}^{\circ} 1$ ).

\section{Cuadro $\mathrm{N}^{0} 1$. Procedencia por departamento de los pacientes que} fallecieron por cáncer de mama. HSF, 2015

\begin{tabular}{|l|c|c|c|}
$\begin{array}{l}\text { Depto. } \\
\text { Comayagua }\end{array}$ & Frecuencia & Porcentaje & $\begin{array}{c}\text { Porcentaje } \\
\text { acumulado }\end{array}$ \\
\hline Cortés & 1 & 7.1 & 7.1 \\
\hline Choluteca & 2 & 14.3 & 21.4 \\
\hline El Paraíso & 1 & 14.3 & 35.7 \\
\hline $\begin{array}{l}\text { Francisco } \\
\text { Morazán }\end{array}$ & 7 & 7.1 & 42.9 \\
\hline Olancho & 1 & 50.0 & 92.9 \\
\hline Total & 14 & 100.0 & 100.0 \\
\hline
\end{tabular}

Fuente: Datos de expedientes. Unidad de Estadística. Hospital San Felipe, 2015

Desde el punto de vista de los municipios, la procedencia de las pacientes evidencia que el municipio del Distrito Central, ocupa el primer lugar con el 50\%; San Pedro Sula y Choluteca, ocupan el segundo lugar con el $14.3 \%$ respectivamente y los municipios de Siguatepeque, Jacaleapa y Catacamas, ocupan el tercer lugar con el 7.1\%, respectivamente (Cuadro $\mathrm{N}^{\circ}$ 2).
Cuadro $\mathrm{N}^{0}$ 2. Procedencia de pacientes por municipios. HSF, 2015

\begin{tabular}{|l|c|c|c|}
\hline $\begin{array}{l}\text { Depto. } \\
\text { Comayagua }\end{array}$ & Frecuencia & Porcentaje & $\begin{array}{c}\text { Porcentaje } \\
\text { acumulado }\end{array}$ \\
\hline Cortés & 2 & 7.1 & 7.1 \\
\hline Choluteca & 2 & 14.3 & 21.4 \\
\hline El Paraíso & 1 & 14.3 & 35.7 \\
\hline $\begin{array}{l}\text { Francisco } \\
\text { Morazán }\end{array}$ & 7 & 7.1 & 42.9 \\
\hline Olancho & 1 & 50.0 & 92.9 \\
\hline Total & 14 & 100.0 & 100.0 \\
\hline
\end{tabular}

La procedencia denota la cercanía de los departamentos y de los municipios al hospital, ya que este último se encuentra en la capital del país. En relación al resto de los municipios, éstos se caracterizan por contar con vías de comunicación adecuadas y con transporte colectivo con variedad de horarios lo que permite la movilización de los pacientes al hospital.

- Edad de las pacientes que fallecieron por cáncer de mama en el HSF

Con respecto a la edad, el grupo de pacientes se encontraba entre el rango de 27 a 85 años. Con una media de 52.7 años; moda de 27 años y mediana de 51. (Cuadro $\mathrm{N}^{\mathrm{o}} 3$ ). El riesgo de desarrollar cáncer de mama, aumenta a medida que la mujer envejece y en la mayoría de los casos, la enfermedad se desarrolla en mujeres mayores de 50 años (http://www.cancer.net/es).

En la casuística que se presenta, el porcentaje acumulado de las mujeres de 50 años y más edad, representa el $56.8 \%$ y el porcentaje acumulado de las mujeres con menos de 50 años, es de $42.6 \%$. La razón entre ambos porcentajes acumulados es de $1 / 1$, por lo que el comportamiento en cuanto a la edad, varía de acuerdo a lo señalado en la referencia internacional.

Cuadro $\mathrm{N}^{0} 3$. Edad de las pacientes que fallecieron por cáncer de mama en el HSF, 2015

\begin{tabular}{|c|c|c|c|}
\hline Edad & Frecuencia & Porcentaje & $\begin{array}{c}\text { Porcentaje } \\
\text { acumulado }\end{array}$ \\
\hline 27 & 1 & 7.1 & 7.1 \\
\hline 34 & 1 & 7.1 & 14.3 \\
\hline 36 & 1 & 7.1 & 21.4 \\
\hline 38 & 1 & 7.1 & 28.6 \\
\hline 40 & 1 & 7.1 & 35.7 \\
\hline 48 & 1 & 7.1 & 42.9 \\
\hline 50 & 1 & 7.1 & 50.0 \\
\hline 52 & 1 & 7.1 & 57.1 \\
\hline 53 & 1 & 7.1 & 64.3 \\
\hline 59 & 1 & 7.1 & 71.4 \\
\hline 67 & 1 & 7.1 & 78.6 \\
\hline 69 & 1 & 7.1 & 85.7 \\
\hline 80 & 1 & 7.1 & 92.9 \\
\hline 85 & 1 & 7.1 & 100.0 \\
\hline Total & 14 & 100.0 & \\
\hline
\end{tabular}

Fuente: expedientes clinicos de pacientes. Unidad de Estadistica. HSF 
- Estado civil de las pacientes que fallecieron por cáncer de mama en el HSF

En relación al estado civil, el $42.9 \%$ de las pacientes se declararon solteras; el grupo de casadas y de unión libre, ocupan el segundo lugar, con el $21.4 \%$ respectivamente y el tercer lugar lo ocupa el estado de viudez con el $14.3 \%$ (Cuadro No 4 ).

Cuadro $\mathrm{N}^{0} 4$. Estado civil de los pacientes que fallecieron por cáncer de mama en el HSF, 2015

\begin{tabular}{|c|c|c|c|}
\hline $\begin{array}{l}\text { Estado } \\
\text { Civil }\end{array}$ & Frecuencia & Porcentaje & $\begin{array}{l}\text { Porcentaje } \\
\text { acumulado }\end{array}$ \\
\hline Casado & 3 & 21.4 & 21.4 \\
\hline Soltero & 6 & 42.9 & 64.3 \\
\hline Viudo & 2 & 14.3 & 78.6 \\
\hline Unión libre & 3 & 21.4 & 100.0 \\
\hline Total & 14 & 100.0 & \\
\hline
\end{tabular}

De acuerdo al Dr. Javier Cortés, del Hospital Vall de Hebrón, de Barcelona, España, en un estudio que dirigió y se realizó en Estados Unidos en el período de 1990 y 2010 en el que participaron 550,000 mujeres, las casadas tienen una supervivencia mayor a los cinco años que las no casadas. El estudio explica que las mujeres casadas obtienen un diagnóstico más temprano y la principal conclusión del mismo es que el pronóstico independientemente de cuando se diagnostique el tumor, es mejor en las casadas que en las solteras (http://consalud.es).

La suma de los promedios de las mujeres que no estaban casadas, solteras y viudas, es de $57.2 \%$; en cuanto a las casadas y la unión libre, es de $42.8 \%$. La razón de ambos promedios es de 1:1. En el grupo que se está observando, el comportamiento no responde a los datos internacionales.

- Ocupación de las pacientes que fallecieron por cáncer de mama en el HSF

En cuanto a la ocupación, el $50 \%$ de las pacientes se dedicaban a los oficios domésticos, el $42.9 \%$ se encontraban desempleadas y el $7.1 \%$ tenía negocio propio (Cuadro $\mathrm{N}^{\circ} 5$ ).

Cuadro $\mathrm{N}^{0} 5$. Ocupación de las pacientes que fallecieron por cáncer de mama en el HSF, 2015

\begin{tabular}{|l|c|c|c|}
\hline Ocupación & Frecuencia & Porcentaje & $\begin{array}{c}\text { Porcentaje } \\
\text { acumulado }\end{array}$ \\
\hline $\begin{array}{l}\text { Servicio } \\
\text { doméstico }\end{array}$ & 7 & 50.0 & 50.0 \\
\hline Desempleado & 6 & 42.9 & 92.9 \\
\hline Negocio propio & 1 & 7.1 & 100.0 \\
\hline Total & 14 & 100.0 & \\
\hline
\end{tabular}

Fuente: expedientes clinicos de los pacientes. Unidad de Estadistica. Hospital San Felipe
En relación a condiciones socioeconómicas, la mayoría de las pacientes, reportan una condición de desprotección social, al ser personas sin empleo y dedicadas a oficios domésticos en más del $90 \%$ de las ellas, siendo un número mínimo, los que poseen un negocio propio. El incremento en el riesgo de padecer o morir por esta causa en países pobres o de ingresos medios se relaciona con los cambios en los patrones reproductivos, nutricionales, disminución en la fecundidad, cambios en los estilos de vida y las condiciones de trabajo de las mujeres. El padecimiento se puede presentar en mujeres de cualquier nivel social, económicos y étnico aunque son las mujeres con mayores desventajas sociales y menores recursos, las más vulnerables (Lozano-Asencio Rafael, 2008).

4.2 Aspectos relacionados con la salud entre las pacientes que fallecieron por cáncer de mama en el HSF

- Causa de admisión de las pacientes que fallecieron por cáncer de mama en el HSF

La causa de admisión o ingreso a la sala de hospitalización, fue en primer lugar por la presencia de masa en un $71.4 \%$; disnea y dolor torácico en segundo lugar con $14.3 \%$, mastalgia (dolor en la mama) y dolor torácico con el $7.1 \%$, respectivamente (Cuadro $\mathrm{N}^{\mathrm{o}} 6$ ).

Cuadro $\mathrm{N}^{0} 6$. Causa de admisión de las pacientes que fallecieron por cáncer de mama en el HSF, 2015

\begin{tabular}{|c|c|c|c|}
\hline $\begin{array}{l}\text { Causa de } \\
\text { admisión }\end{array}$ & Frecuencia & Porcentaje & $\begin{array}{l}\text { Porcentaje } \\
\text { acumulado }\end{array}$ \\
\hline Masa & 10 & 71.4 & 71.4 \\
\hline Mastalgia & 1 & 71.1 & 78.6 \\
\hline Tinte ictérico & 1 & 71.1 & 85.7 \\
\hline $\begin{array}{l}\text { Disnea y dolor } \\
\text { torácico }\end{array}$ & 2 & 14.3 & 100 \\
\hline Total & 14 & 100.0 & \\
\hline
\end{tabular}

- Pacientes que presentaron menarquia antes de los once años entre las que fallecieron por cáncer de mama en el $H S F$

Los datos muestran que el $85.7 \%$ de las pacientes presentaron la menarquia en edades posteriores a los once años y el $14.3 \%$ la presentó antes de los once años (Cuadro $\mathrm{N}^{\circ} 7$ ).

Cuadro $\mathrm{N}^{0} 7$. Pacientes que presentaron menarquia antes de los once años entre las que fallecieron por cáncer de mama en el HSF, 2015

\begin{tabular}{|c|c|c|c|}
$\begin{array}{c}\text { Menarquia } \\
\text { antes de los } \\
\text { 11 años }\end{array}$ & Frecuencia & Porcentaje & $\begin{array}{c}\text { Porcentaje } \\
\text { acumulado }\end{array}$ \\
\hline SI & 2 & 14.3 & 14.3 \\
\hline NO & 12 & 85.7 & 100.0 \\
\hline Total & 14 & 100.0 & \\
\hline
\end{tabular}

Fuente: expedientes clinicos. Unidad de Estadistica. Hospital San Felipe, 2015 
Las mujeres que hayan tenido más ciclos menstruales debido a que comenzaron a menstruación a una edad temprana, antes de los 11 años, presentan un riesgo ligeramente mayor de padecer cáncer de mama. Este aumento en el riesgo podría deberse a una exposición más prolongada a las hormonas estrógeno y progesterona durante la vida producidos por los ovarios (www.aecc.es/ SOBREELCANCER).

Brinton y colaboradores, examinaron los factores reproductivos en uno de los estudios más trascendentes y reportaron que las mujeres que iniciaron la menstruación antes de los 12 años tenían riesgo relativo de 1.3 de cáncer invasor comparado con las que iniciaron después de los 15 años (Brinton, Schairer, Hoover, \& Fraumeni, 1988). Paredes reportó la relación significativa del cáncer de mama y menarquia temprana (Paredes, 1991).

- Antecedentes de menopausia de las pacientes que fallecieron por cáncer de mama en el HSF

Los pacientes mostraron antecedentes de menopausia al momento de su ingreso en un $64.3 \%$; no presentaban la menopausia en un $28.6 \%$, no aparece consignado en la historia clínica, en un $7.1 \%$ (Cuadro $\left.\mathrm{N}^{\circ} 8\right)$.

Cuadro $\mathrm{N}^{\circ} 8$. Antecedentes de menopausia de las pacientes que fallecieron por cáncer de mama en el HSF, 2015

\begin{tabular}{|c|c|c|c|}
$\begin{array}{c}\text { Pacientes } \\
\text { con } \\
\text { menopausia }\end{array}$ & Frecuencia & Porcentaje & $\begin{array}{c}\text { Porcentaje } \\
\text { acumulado }\end{array}$ \\
\hline SI & 9 & 64.3 & 64.3 \\
\hline NO & 4 & 28.6 & 92.9 \\
\hline $\begin{array}{c}\text { No apacere } \\
\text { consignado }\end{array}$ & 1 & 7.1 & 100.0 \\
\hline Total & 14 & 100.0 & \\
\hline
\end{tabular}

Fuente: datos de los expedientes de los pacientes. Unidad de Estadistica. Hospital San Felipe, 2015

Estudios muestran que tras la menopausia la fuente de estrógenos se encuentra en la glándula suprarrenal con una modificación en el tejido graso de la hormona que se produce en esta glándula y que se traduce a estrógenos (estradiol y estrona). Se considera que la presencia de menopausia después de los 55 años, aumenta el riesgo(www.aecc.es).

El cáncer de mama tiene su mayor incidencia en esta etapa de la vida de la mujer, aunque se observa un descenso progresivo a partir de la menopausia. La menopausia precoz es un factor protector de padecer cáncer de mama. Una mujer con una menopausia natural a los 45 años tiene la mitad de riesgo de padecer de cáncer de mama que la mujer que tiene la menopausia a los 55 años. La extirpación de los ovarios, tiene factor protector en las mujeres que tienen predisposición genética de alto riesgo (en los genes BRCA1 y BRCA2) (www.aecc.es/sobreelcancer).
- Factores de riesgo personal y familiar entre las pacientes que fallecieron por cáncer de mama en el $H S F$

Los datos de las pacientes, relacionados con factores de riesgo familiar muestran que tanto los antecedentes personales de cáncer de mama como la presencia de parientes en primer grado con esta patología, resultó negativa en un $100 \%$ (Cuadro $\mathrm{N}^{\circ}$ 9).

Cuadro $\mathrm{N}^{0} 9$. Factores de riesgo personal y familiar. HSF, 2015

\begin{tabular}{|c|c|c|c|}
\multicolumn{2}{|c|}{$\begin{array}{c}\text { Antecedentes personales } \\
\text { de cáncer de mama }\end{array}$} & \multicolumn{2}{|c|}{$\begin{array}{c}\text { Parientes en primer grado } \\
\text { con cáncer de mama }\end{array}$} \\
\hline NO & $85.71 \%$ & NO & $92.85 \%$ \\
\hline SI & $14.28 \% *$ & SI & $7.14 \%$ \\
\hline
\end{tabular}

* Dos pacientes con cáncer en una de sus mamas y posteriormente en la otra

Fuente: datos tomados de expedientes clinico. Unidad de Estadistica. Hospital San Felipe. 2015

La predisposición hereditaria al cáncer de mama en las mujeres jóvenes se ha documentado ampliamente y en estudios realizados se encontró que el antecedente familiar de cáncer de mama predice la probabilidad de desarrollarlo. La carga genética de la enfermedad está asociada con un aumento de 2 veces en la incidencia por este tumor y el riesgo se incrementa, si más de un familiar ha padecido la enfermedad o si algún miembro de la familia la padeció en edades tempranas, es decir, antes de la menopausia (Olaya, Pierre, Lazcano, Villamil, \& Posso, 1999).

- Uso de anticonceptivos y consumo de alcohol y tabaco entre las pacientes que fallecieron por cáncer de mama en el HSF

Los datos muestran que la utilización de anticonceptivos y el consumo de alcohol y tabaco, tienen un dato negativo en un $100 \%$ (Cuadro $\mathrm{N}^{\mathrm{o}} 10$ ).

Cuadro $\mathrm{N}^{0} 10$. Antecedentes de uso de anticonceptivos, consumo de alcohol y de tabaco pacientes que fallecieron por cáncer de mama. HSF, 2015

\begin{tabular}{|c|c|c|c|c|c|}
\multicolumn{2}{c|}{$\begin{array}{c}\text { Uso de } \\
\text { anticonceptivos }\end{array}$} & \multicolumn{2}{c|}{$\begin{array}{c}\text { Consumo de } \\
\text { alcohol }\end{array}$} & \multicolumn{2}{|c|}{$\begin{array}{c}\text { Consumo } \\
\text { de tabaco }\end{array}$} \\
\hline NO & $100 \%$ & NO & $100 \%$ & NO & $100 \%$ \\
\hline
\end{tabular}

Fuente: datos tomados de expedientes clínico. Unidad de Estadística. Hospital San Felipe. 2015

Estudios realizados, mencionan que las mujeres que fuman, ingieren alimentos con alto contenido graso, beben alcohol y no hacen suficiente ejercicio corren un mayor riesgo de desarrollar cáncer de mama (Sánchez \& Verga, 2011). Otros estudios, especifican que podrían evitarse hasta el 14\% de los casos de cáncer si se redujera en forma considerable el consumo nocivo de alcohol o se eliminará. El uso prolongado del tratamiento hormonal sustitutivo 
después de la menopausia se ha asociado a un mayor riesgo de padecer cáncer de mama.

El uso de anticonceptivos orales (pastillas anticonceptivas) parece aumentar levemente el riesgo de aumentar cáncer de mama, pero solo durante un período limitado. Las mujeres que hayan dejado de consumir anticonceptivos orales por más de 10 años, no parecen presentar mayor riesgo de desarrollar cáncer de mama (OPS/OMS, 2014).

- Paridad entre las pacientes que fallecieron por cáncer de mama en el HSF

Los datos de paridad muestran que las pacientes con cuatro hijos representan el primer lugar con $28.6 \%$; la paridad de más de 6 hijos, 5 hijos, un hijo y cero hijos, representa el $14.3 \%$. La paridad de 2 y 3 hijos representan el $7.1 \%$ (Cuadro $\mathrm{N}^{\mathrm{o}} 11$ ).

Cuadro $\mathrm{N}^{0} 11$. Paridad entre las pacientes que fallecieron por cáncer de mama en el HSF, 2015

\begin{tabular}{|c|c|c|c|}
\hline $\mathbf{N}^{0}$ de hijos & Frecuencia & Porcentaje & $\begin{array}{l}\text { Porcentaje } \\
\text { acumulado }\end{array}$ \\
\hline 0 hijos & 2 & 14.3 & 14.3 \\
\hline 1 hijo & 2 & 14.3 & 28.6 \\
\hline 2 hijos & 1 & 7.1 & 35.7 \\
\hline 3 hijos & 1 & 7.1 & 42.9 \\
\hline 4 hijos & 4 & 28.6 & 71.4 \\
\hline 5 hijos & 2 & 14.3 & 85.7 \\
\hline $\begin{array}{l}\text { Más de } 5 \\
\text { hijos }\end{array}$ & 2 & 14.3 & 100 \\
\hline Total & 2 & 14.3 & \\
\hline
\end{tabular}

Respecto a las condiciones reproductivas y el cáncer de mama, más de la mitad de las pacientes tuvieron una paridad importante, habiendo presentado un patrón de multiparidad con más de 3 hijos, lo que contradice lo establecido a nivel internacional en diferentes artículos que define a la paridad como un factor protector de esta patología.

De hecho estos estudios mencionan que el embarazo temprano y el incremento en la paridad, son factores protectores de cáncer de mama (Nechuta, Paneth, \& Velie, Pregnancy characteristics and maternal breast cancer risk: a review of the epidemiologic literature, 2010). (OlayaContreras, Buekens, Lazcano-Ponce, Villamil-Rodríguez, \& Posso-Valencia, 1999). Sin embargo, otros estudios mencionan que el mecanismo biológico entre el rol que desempeña el embarazo en el cáncer de mama, no tiene una etiología muy clara, pero algunas hipótesis han sido propuestas, cada una de las cuales, establecen el rol positivo de las hormonas durante el embarazo (Nechuta, Paneth, \& Velie, 2010).

- Pacientes que se realizaron el examen de mamografia entre las mujeres que fallecieron por cáncer de mama en el HSF

En un parámetro específico de acceso a los servicios de salud, se logra evidenciar que se realizaron la mamografía el $21.4 \%$; y no lograron realizarla el $78.6 \%$. En cuanto a la realización de la biopsia, el $100 \%$ de las pacientes lo lograron. La realización de quimioterapia lo lograron la mitad de las pacientes. La realización de la mastectomía se llevó a cabo en el 43\% de los pacientes, pero no llegaron a la misma el 57\% y en cuanto a la radioterapia, la lograron únicamente el $21.42 \%$ de las pacientes (Cuadro $\mathrm{N}^{\mathrm{o}} 12$ ).

Cuadro $\mathrm{N}^{0} 12$. Acceso a procedimientos y tratamientos de pacientes que fallecieron de cáncer de mama. HSF, 2015

\begin{tabular}{|c|c|c|c|c|c|c|c|c|c|}
$\begin{array}{c}\text { Realizaron } \\
\text { mamografía }\end{array}$ & $\begin{array}{c}\text { Realizaron } \\
\text { biopsia }\end{array}$ & \multicolumn{2}{c|}{$\begin{array}{c}\text { Realizaron } \\
\text { quimioterapia }\end{array}$} & \multicolumn{2}{c|}{$\begin{array}{c}\text { Realizaron } \\
\text { mastectomia }\end{array}$} & \multicolumn{2}{c|}{$\begin{array}{c}\text { Realizaron } \\
\text { radioterapia }\end{array}$} \\
\hline SI & $21.4 \%$ & SI & $100 \%$ & SI & $50 \%$ & SI & $42.85 \%$ & SI & 21.42 \\
\hline NO & $78.6 \%$ & NO & $0 \%$ & NO & $50 \%$ & NO & $57.14 \%$ & NO & $78.57 \%$ \\
\hline Total & $100 \%$ & Total & $100 \%$ & Total & $100 \%$ & Total & $100 \%$ & Total & 100.0 \\
\hline \multicolumn{2}{|c|}{ Fuente: datos tomados de expedientes clinico. Unidad de Estadistica de Hospital San Felipe, 2015} \\
\hline
\end{tabular}

La OMS señala que la mamografía es el único método de cribado que se ha revelado que sea eficaz, si su cobertura supera el $70 \%$. Esta forma de cribado puede reducir la mortalidad por cáncer de mama en un 20\%-30\% en las mujeres de más de 50 años en los países de ingresos altos. El tamizaje basado en esta técnica es muy complejo $\mathrm{y}$ absorbe muchos recursos y no se ha hecho ninguna investigación sobre su eficacia en los entornos con recursos escasos, es decir en los países con ingresos medios/bajos, como Honduras (http://www.who.int).

Es importante destacar que todas las pacientes tuvieron la oportunidad que se le realizara una biopsia con la cual se estableció el diagnóstico definitivo del cáncer (http://www.cancer.net/es). En relación a la quimioterapia, su utilización depende del tipo de cáncer y de cuando se haya diseminado el tumor (http://www.cancer.net/es). La cirugía, es el tipo más antiguo de terapia contra el cáncer y en la actualidad sigue siendo un tratamiento eficaz para muchos tipos de cáncer (http://www.cancer.net/es).

En el estudio, menos de la mitad tuvieron la oportunidad de intervención quirúrgica. La radioterapia se utiliza como principal tratamiento contra el cáncer y para otro tipo de tratamiento. Se administra después del tratamiento principal para atacar las células cancerosas restantes (http://www.cancer.net/es). 
En el estudio, menos del tercio de las pacientes, tuvieron la oportunidad de este tipo de tratamiento.

- Letalidad entre las pacientes que fallecieron por cáncer de mama en el HSF

La tasa de letalidad específica para cáncer de mama, es de $0.80 \%$, expresando el peso específico de la misma en el grupo de pacientes que fallecieron en el año 2015 en el hospital.

La tasa de letalidad hospitalaria por cáncer de mama permite observar el grado de agresividad de la enfermedad a través del número de personas fallecidas por ésta en relación al total de egresos hospitalarios. Establece la calidad de los servicios para el ingreso específico de pacientes a una institución.

Datos suministrados por el INEGI (Instituto Nacional de Estadística y Geografía) de México para el año 2013, muestran que la media de letalidad hospitalaria para cáncer de mama por diferentes edades, se ha sostenido por tres años con valores similares a 4 por 100 egresos (INEGI, 2015). En el caso del Hospital San Felipe, el dato es menor, siendo de aproximadamente 1 por cada 100 egresos, es decir, 4 veces menos. De acuerdo a lo establecido, la letalidad es un indicador de la calidad de los servicios hospitalarios que se prestan en el hospital. También está relacionado con las condiciones propias de la enfermedad de las pacientes.

- Tasa bruta de mortalidad entre las pacientes que fallecieron por cáncer de mama en el HSF

La tasa bruta de mortalidad (menos de 48 horas), muestra que, en el año 2015, es de $0.014(3 / 20,346)$ y expresa que, del total de cien egresos, el riesgo de muerte es de menos de $0.01 \%$ para el hospital en el período observado (Cuadro $\mathrm{N}^{\circ} 13$ ).

Cuadro $\mathrm{N}^{0} 13$. Tasa bruta de mortalidad. HSF, 2015

\begin{tabular}{|l|c|c|c|}
\hline Servicios & Fallecidos & Egresos & Tasa \% \\
\hline Oncología & 0 & 2,327 & 0 \\
\hline Cirugías & 0 & 2,311 & 0 \\
\hline Medicinas & 0 & 855 & 0 \\
\hline Oftalmología & 0 & 612 & 0 \\
\hline Pediatría & 1 & 1,956 & 0,051 \\
\hline Maternidad & 2 & 12,277 & 0,016 \\
\hline Asilo & 0 & 8 & 0 \\
\hline Total hospital & 3 & 20,346 & 0.014 \\
\hline
\end{tabular}

Fuente: datos obtenidos de la Unidad de Estadística del HSF, 2015

El dato denota que, a nivel de todo el hospital, la mortalidad total por todos los egresos que se suscitaron en el centro para el año 2015 es baja, tomando en cuenta que las muertes se relacionan con las condiciones de ingreso del paciente y condiciones de compromiso de la vida misma del paciente. Para este período, el dato es positivo para el hospital en vista de que la tasa es menor a 10 por 100 egresos (estándar limite a nivel de país). Para el caso que nos ocupa, el servicio de Oncología no muestra muertes acaecidas antes de las 48 horas lo que resulta un aspecto muy positivo.

- Tasa neta de mortalidad entre las pacientes que fallecieron por cáncer de mama en el HSF

La tasa neta de mortalidad del Hospital San Felipe para el año 2015, es de 3.11 y expresa que del total de cien egresos (muertes) acaecidas a partir de los ingresos realizados por el hospital de más de 48 horas, el riesgo de muerte es de 3. En forma particular y nivel de los servicios de salud, el que presenta mayor tasa neta de mortalidad son las áreas de medicina interna con aproximadamente 7 por cada cien egresos. Posteriormente, se encuentra el servicio de oncología con aproximadamente 5 por cien egresos y el servicio de cirugía que reporta para ese período en observación, menos de $0.5 \%$ (Cuadro $\mathrm{N}^{\circ} 14$ ).

Cuadro $\mathrm{N}^{0}$ 14. Tasa neta de mortalidad, HSF. 2015

\begin{tabular}{|l|c|c|c|}
\hline Servicios & Fallecidos & Egresos & Tasa \% \\
\hline Medicina & 59 & 855 & 6.90 \\
\hline Oncología & 109 & 2,327 & 4.68 \\
\hline Cirugía & 3 & 2,311 & 0.12 \\
\hline Total Hospital & 171 & $5,493^{*}$ & 3.11 \\
\hline
\end{tabular}

Fuente: Unidad de Estadistica de Hospital San Felipe, 2015

*Incluye los datos de las muertes de los servicios que las informaron

La tasa neta de mortalidad es una medida indirecta de la capacidad resolutiva del hospital y tienen que ver con la gestión de recursos para la población a atender como también con la capacidad técnica que se oferta.

Es importante denotar que tiene que ver con las condiciones previas de estado de salud del paciente. La tasa específica para el servicio de oncología es baja y el resultado es positivo para el hospital, ya que es menor de 10, el que representa el referente de calidad.

4.3 Años de vida potencialmente perdidos tomando como base la esperanza de vida hasta el año 2014

Los años de vida potencialmente perdidos, expresa el número de años que dejan de vivir las personas al momento de morir de tal forma que refleja la mortalidad prematura, es decir, el impacto de la mortalidad en los grupos más jóvenes. Los datos muestran que, por la causa específica de cáncer de mama, el grupo de mujeres observadas perdieron 379 años, ya que murieron en forma prematura, ajustado por la esperanza de vida, para esta causa (Cuadro $\mathrm{N}^{\mathrm{o}} 15$ ). 
Cuadro $\mathrm{N}^{0} 15$. Años potencialmente de vida perdidos por cáncer de mama. HSF, 2015

\begin{tabular}{|c|c|c|c|c|}
\hline $\begin{array}{c}\text { Grupos de } \\
\text { edad }\end{array}$ & Frecuencia (f) & $\begin{array}{c}\text { Punto medio } \\
\text { del grupo de edad }\end{array}$ & $\begin{array}{c}\text { APVP individual } \\
\sum(\text { L-Xi) }\end{array}$ & $\begin{array}{c}\text { APVP por grupo de } \\
\text { edad } \sum \text { (L-Xi)f }\end{array}$ \\
\hline $25-29$ & 1 & 27 & 52 & 52 \\
\hline $30-34$ & 1 & 32 & 47 & 47 \\
\hline $35-39$ & 2 & 37 & 42 & 84 \\
\hline $40-44$ & 1 & 42 & 37 & 37 \\
\hline $45-49$ & 1 & 47 & 32 & 32 \\
\hline $50-54$ & 3 & 52 & 27 & 81 \\
\hline $55-59$ & 1 & 57 & 22 & 22 \\
\hline $65-69$ & 2 & 67 & 12 & 24 \\
\hline Total & $12^{*}$ & & 271 & 379 años \\
\hline
\end{tabular}

Fuente: Unidad de Estadistica de Hospital San Felipe, 2015

*Se excluyen los dos casos que murieron en edades mayores a la esperanza de vida de país para el año 2014 (79 años)

Al no contar con otro estudio similar en el mismo hospital, el total de años perdidos por esta causa permite establecer que tanto el acceso como la calidad de atención del servicio y la oportunidad del abordaje, han disminuido la mortalidad en edades precoces, es decir, antes del límite superior de la esperanza de vida establecida para el país para el período del estudio.

En este estudio se logra establecer que el grupo de 35 a 39 años, es el que más años de vida perdieron, mostrando el mismo comportamiento el grupo de 50-54 años. El grupo que menos años de vida perdió, es el grupo de edad de 55 a 59 años. Estos datos obligan a realizar con mayor profundidad la indagatoria de factores de riesgo, que permitan establecer las causas por las cuales se observa este comportamiento. De acuerdo a estudios realizados en otros países, los AVPP por cáncer de mama representan más de $1 / 3$ de las muertes por esta neoplasia, en edades tempranas, es decir, antes de los 50 años, tal y como ocurre con las pacientes del Hospital San Felipe, por lo que requiere que su detección y tratamiento lleguen oportunamente y se utilicen estrategias de atención que cubran las diferentes necesidades y demandas en la materia en estos grupos de edad (Agudelo, Aguirre, \& Dávila, 2010).

\section{Referencia final}

La población mundial se encuentra en un proceso de envejecimiento creciente lo que implica el enfrentar múltiples retos en el sector salud, uno de ellos es lograr establecer la relación de este proceso con el incremento de casos de cáncer en los países en vías de desarrollo. En el año 2014, la Organización Mundial de la Salud, reportó la distribución de mortalidad por cáncer y en el caso específico de cáncer de mama en mujeres, el dato fue de $8.2 \%$ (OMS, 2014).

Considerando que el cáncer de mama se ha convertido en uno de los problemas de salud de mayor connotación en el grupo femenino, en el estudio, se ha logrado documentar factores de riesgo relacionados con la enfermedad, así como el acceso a los servicios de salud, la oportunidad de abordaje y los años de vida potencialmente perdidos por esta causa a nivel hospitalario. Al indagar en los datos de las pacientes, se observa que, desde el punto de vista geográfico, la mitad de las pacientes procedían del municipio del Distrito Central que incluyen a la ciudad de Tegucigalpa y Comayagüela, (ambas de Honduras).

A su vez, se establece en la casuística, la presencia de pacientes que pertenecen a municipios que se encuentran en la zona norte y sur, que cuentan con sistemas de comunicación a través de carretera en condiciones adecuadas y transporte que viabiliza el acceso para llegar al centro de hospitalización, así como municipios que se encuentran en el oriente del país, que presentan similares características, lo que le ofrece a los pacientes la oportunidad de la hospitalización, pero la muerte de las mismas. Como resultado de la salud, obliga a indagar aspectos que profundicen aún más en el acceso y la utilización de los servicios de salud por parte de la población con desventaja social. Datos probatorios a nivel de la OMS muestran que los más pobres tienen la peor salud. Este es un fenómeno mundial que se presenta en los países de ingresos bajo, mediano y altos. Los datos dentro de los países indican que, en general cuanto más baja es la posición socioeconómica de una persona, peor es su salud. A esto se le conoce como gradiente social de la salud (WHO, 2011).

En relación a condiciones socioeconómicas, la mayoría de las pacientes, reportan una condición de desprotección social, al ser personas sin empleo y dedicadas a oficios domésticos en más del $90 \%$ de las ellas, siendo un número mínimo, los que poseen un negocio propio. $\mathrm{El}$ incremento en el riesgo de padecer o morir por esta causa en países pobres o de ingresos medios se relacionan con los cambios en las patrones reproductivos, nutricionales, disminución en la fecundidad, cambios en los estilos de vida y las 
condiciones de trabajo de las mujeres (Lozano-Asencio Rafael, 2008).

Destaca el hecho de que estas condiciones sociales son las que obstaculizan la realización de exámenes cribados, como el caso de la mamografía, ya que en los datos que reporta la OMS, relacionan este método diagnóstico como efectivo en más de un $70 \%$ pero en países con ingresos altos. Aún no hay estudios que relacionan la eficacia de la mamografía con países con ingresos medios/bajos como Honduras, por lo que cobra mayor importancia la introducción de actividades de detección temprana en los servicios de atención primaria que consideren aspectos culturales, financieros, así como la prestación de servicios de salud mamaria que sean adecuados y eficientes. Esto requiere que se capacite al personal y se eduque a la población. También es importante destacar la relación con estilos de vida, los hábitos de fumar y consumir bebidas alcohólicas, que en el caso de la población estudiada estos datos resultaron negativos en un $100 \%$ en las pacientes.

Respecto a las condiciones reproductivas y el cáncer de mama, más de la mitad de las pacientes tuvieron una paridad importante, habiendo presentado un patrón de multiparidad con más de 3 hijos, lo que contradice lo establecido a nivel internacional en diferentes artículos que define a la paridad como un factor protector de esta patología. De hecho, estos estudios mencionan que el embarazo temprano y el incremento en la paridad, son factores protectores de cáncer de mama (Nechuta, Paneth, \& Velie, 2010). (Olaya-Contreras, Buekens, LazcanoPonce, Villamil-Rodríguez, \& Posso-Valencia, 1999).

Los factores de riesgo por poblaciones pueden ser modificables y con lo mencionado anteriormente se puede evidenciar que se debe intensificar el trabajo relacionado con los servicios de salud y los programas de salud comprometidos con esta patología con el objetivo de lograr investigar con mayor profundidad y definir en forma específica la población hacia la cual se enfocaran todos los esfuerzos en nuestra población.

De acuerdo a la OMS (OMS, 2007), para que la detección temprana sea efectiva, una de las estrategias es concientizar a la paciente con respecto a los primeros signos y síntomas y en ese sentido, la autoexploración es la actividad más relevante de esta estrategia. Sin embargo, en ninguna de las historias clínicas, se encuentra consignada la misma.

La autoexploración mamaria no ha demostrado un efecto en la reducción de la mortalidad pero tiene la capacidad de detectar abultamientos desde 0.5 a $1.0 \mathrm{cms}$ (Cárdenas, Gonzáles, \& Santiago, 2006).

Los datos revelan que el motivo de la consulta rescata el hecho que el $80 \%$ de las pacientes fueron hospitalizadas por la presencia de masas y de mastalgia. Esto obligaría que el interrogatorio al respecto sea más exhaustivo para lograr establecer con el mismo el proceso de oportunidad de las pacientes de acceder en forma temprana a los servicios de salud al encontrarse alguna masa pequeña o dolor en la mama. Sería imprescindible conocer durante el interrogatorio el nivel de información que las pacientes tienen al respecto, ya que ante la inexistencia en el país de programas de educación en salud o promoción de salud en forma continua y a edades tempranas no se logra alcanzar en la población el nivel de consciencia que se pretende y que sea efectiva.

Otra estrategia en la detección temprana, es el tamizaje a nivel nacional por medio de la mamografía a partir de los 50 años hasta los 65 (en caso de antecedentes familiares de cáncer de mama, la mastografía se realizará a partir de los 45 años de edad) (Santana-Chávez, ZepedaFlores, González-Muñoz, \& Meñendez- Morales, 2015). Sin embargo, la red hospitalaria estatal no cuenta con mamógrafos como parte de la tecnología que se debe ofrecer a la población femenina. La oportunidad a través de esta otra vía se vuelve limitante o nula, considerando las condiciones económicas- sociales de las pacientes observadas en este estudio, lo que explicaría las razones del porque en aproximadamente $80 \%$ de las pacientes no se realizó este análisis.

La Organización Mundial de la Salud ( 2007), señala que la segunda fase por la que se deben esforzar los servicios de salud, es la fase del tratamiento, que pretende curar la enfermedad y alargar la vida. Sin embargo, en el caso del grupo de pacientes que nos ocupa, la oportunidad de abordaje del paciente únicamente se cumplió con la realización de la biopsia, la que se logró en un $100 \%$. Es relevante considerar el hecho que las pacientes tienen este servicio por parte del Estado, lo que le permite tener cierta oportunidad de realización. Sin embargo, con respecto al tratamiento, únicamente el $50 \%$ tuvieron la oportunidad de la quimioterapia, siendo menores los porcentajes para la radioterapia y la mastectomía.

Al relacionar la tasa neta de mortalidad en el hospital en el año 2015, con datos a nivel internacional, la que fue de 3 por 100 egresos y la tasa de letalidad de cáncer de mama, para este período la que fue de 1 por 100 egresos, define el peso específico que tiene esta causa de muerte en la medición de la mortalidad para el hospital para este período de tiempo, sumado el resultado que brindan los años de vida potencialmente perdidos, con lo que se evidencia la carga de enfermedad que esta causa representa en relación a las muertes prematuras tomando como parámetro la esperanza de vida.

En un país, con condiciones tan precarias sociales y económicas, debe destacarse que la detección temprana del cáncer de mama, requiere el diagnóstico precoz por parte 
de las mujeres, ya que este mejoraría la supervivencia, disminuiría la morbilidad, reduciría los costos de atención si se diagnostica con prontitud y se trataría en forma eficaz (Martíne-Sánchez, Escudero de los Ríos, Arias-Flores, \& Barrios-Bautista, 2013). Para lograr lo anterior y con toda la modificación que se está realizando, del modelo de atención, se deben brindar las condiciones para que las pacientes tengan conciencia del problema de salud que representa el cáncer de mama.

Sería imprescindible, iniciar programas de educación para la salud, en forma masiva que incluya el tamizaje por ECM (examen clínico de mama, que se inicia por el autoexamen). Para lograr una meta efectiva, se debería brindar esta información en todas las unidades de salud del país, tanto pública como privada, ya que un punto relevante, es que, desde el punto de vista geográfico, se evidencian en esta casuística únicamente 6 de 18 departamentos con condiciones de comunicación terrestre y aun así, las pacientes murieron. La interrogante se realiza con las pacientes que viven en otras áreas geográficas que no cuentan con vías de acceso. Se requiere, por lo tanto, realizar alianzas estratégicas con diferentes actores que logren ofertar diferentes formas de atención, siendo la prioritaria, la preventiva y para realizarlo, es importante la identificación de poblaciones metas, es decir, un plan nacional, local y descentralizado muy bien articulado. En el contexto de esta planificación y en relación a este estudio, destaca el hecho que el grupo de edad que más impacta en cuanto APVP es el de 35 a 39 años, ocupando el segundo lugar el de 50 a 54 años. En ese sentido, la literatura señala que la edad de riesgo aumenta con la edad y la mayor incidencia se encuentra entre 50 y 65 años, por lo que la prematurez que se evidencia en este estudio en cuanto a edad, debe ser objeto de priorización. Es necesario visualizar aspectos relevantes para que se vuelvan más eficaces las acciones con respecto al cáncer de mama en Honduras.

\section{Bibliografía}

- Agudelo, M., Aguirre, A., \& Dávila, C. (2010). Variaciones en los años de vida perdidos por cánceres de mama y cervico uterino en México según grado de marginación estatal, 1997 y 2007. Distrito Federal: Rev. Chil Salud Pública 2010; Vol 14 (1): 8-17.

- BID, OPS, \& Mundial, B. (2001). La salud de la mujer en América Latina y el Caribe. Washington.

- Brinton, L., Schairer, C., Hoover, R., \& Fraumeni, J. (1988). Menstrual factor and risk of breast cancer. Cancer Invest 1988, 6(3): 245-54.

- Cárdenas, L., Gonzáles, N., \& Santiago, S. (2006). Prevención, tamizaje y referencia oportuna de casos sospechosos de cáncer de mama en el pimer nivel de atención.
- Del Valle Gómez María Olivo, L. G. (1993). Análisis de los años potenciales de Vida perdidos por cáncer en Asturias y España. Oviedo: Rev San Hig Pub 1993; 67 : 129-144.

- Gran, M., \& Torres, R. (2010). Indicadores Básicos para el Análisis del Estado de Salud de la Población. La Habana.

- http://consalud.es/empresas/empresas-con-salud/elestado-civil-un-nuevo-factor-atener-en-cuenta-en-elpronostico-del-cancer-de-mama-11048. (s.f.).

- http://www.breastcancer.org/es/sintomas/cancer_de mama/riesgo/factores. (s.f.).

- http://www.cancer.net/es/desplazarse-poratenci $\% \mathrm{C} 3 \% \mathrm{~B} 3 n$-delc $\% \quad \mathrm{C} 3 \% \mathrm{~A} 1$ ncer $/ \mathrm{c} \% \mathrm{C} 3 \% \mathrm{~B} 3 \mathrm{mo}-$ se-trata-elc $\% \mathrm{C} 3 \% \mathrm{~A} 1 \mathrm{ncer} / \mathrm{cirug} \% \mathrm{C} 3 \% \mathrm{ADa} /$ qu $\%$ C $3 \%$ A 9 -es-1a-cirug $\%$ C $3 \%$ ADaonco $1 \%$ $\mathrm{C} 3 \% \mathrm{~B} 3$ gica. (s.f.).

- http://www.cancer.net/es/desplazarse-poratenci $\% \mathrm{C} 3 \% \mathrm{~B} 3 \mathrm{n}$-delc $\% \mathrm{C} 3 \% \mathrm{~A} 1 \mathrm{ncer} / \mathrm{c} \% \mathrm{C} 3 \% \mathrm{~B} 3 \mathrm{mo}-$ se-trata-el-c\% C 3\% A 1 ncer/quimioterapia/ qu $\% \mathrm{C} 3 \% \mathrm{~A} 9$-es-la-quimioterap. (s.f.).

- http://www.cancer.net/es/tipos-de-c\%C3\%A1ncer/ c\%C3\%A1ncer-demama/diagn $\% \mathrm{C} 3 \% \mathrm{~B} 3$ stico. (s.f.).

- http://www.cancer.net/es/tipos-de-c\%C3\%A1ncer/ c\%C3\%A1ncer-de-mama/factores-deriesgo. (s.f.).

- http://www.who.int/topics/cancer/breastcancer/es/ index3.html. (s.f.).

- INEGI. (2015). Estadísticas a propósitos del día mundial de la lucha contra el cáncer de mama.

- Knaul, F., Bustreo, F., Ha, E., \& Langer, A. (2009). Breast cancer: Why link early detection to reproductive health interventions in developing countries? Salud Pública de México/ Vol. 51, suplemento 2 de 2009.

- Lozano-Asencio Rafael, G.-D. H.-S.-C. (2008). Tendencias del cáncer de mama en América Latina y el Caribe. Washington.

- M., D. (1947). Decline in tuberculosis: the death rate fails to tell the entire story. Am Rev Tubercul 1947; 86: 157-64.

- Martíne-Sánchez, Y., Escudero de los Ríos, P., AriasFlores, R., \& Barrios-Bautista, F. (2013).

- Epidemiología del cáncer en pacientes adultos del Hospital de Oncología de Centro Médico Siglo XXI, Instituto Mexicano del Seguro Social. México, D.F.

- Nechuta, S., Paneth, N., \& Velie, E. (2010). Pregnancy characteristics and maternal breast cancer risk: a review of the epidemiologic literature. NIH Public Access. Cancer Causes Control. 2010 July; 21(7).

- Nechuta, S., Paneth, N., \& Velie, E. (2010). Pregnancy characteristics and maternal breast cancer risk: a review of the epidemiological literature. NIH Public Acces. 
- Olaya, P., Pierre, B., Lazcano, E., Villamil, J., \& Posso, H. (1999). Factores de riesgo reproductivo asociados al cancer mamario en mujeres colombianas.

- Olaya-Contreras, P., Buekens, P., Lazcano-Ponce, E., Villamil-Rodríguez, J., \& Posso-Valencia, H. (1999). Factores de Riesgo reproductivo asociados al cáncer mamarioen mujeres colombianas. Journal of Public Health. Vol 33. No.3. Junio 1999.

- OMS. (2004). Reporte de Salud 2004. Ginebra.

- OMS. (2007). Control de cáncer. Aplicación de los conocimientos. Guía de la OMS para desarrollar programas eficaces. Política y abogacía. Ginebra.

- OMS. (2014). Perfiles de cáncer 2014.

- OMS. (2014). Perfiles oncológicos de los países. 2014. Ginebra.

- OPS, B. (2003). Técnicas para la medición del impacto de la mortalidad: Años potenciales de vida perdidos. Washington: Boletín. Vol. 24. No.2.

- OPS/OMS. (2014). Prevención: factores de riesgo y prevención del cáncer de mama.

- Paredes, A. (1991). Factores de riesgo en cáncer mamario . Gynecol Obstet Mex 1991;59:49-55.

- Sánchez, J., \& Verga, B. (2011). Cáncer de mama: es posible prevenirlo? Rev. Ciencias Médicas.eneromarzo,2011;15(1):14-28.

- Santana-Chávez, L., Zepeda-Flores, S., GonzálezMuñoz, L., \& Meñendez-Morales, J. (2015).

- SESAL. (2010). Indicadores Básicos para el Análisis del Estado de Salud de la población.

- SESAL. (2014). Anuario Estadístico Año 2014. Tegucigalpa.

- Toro Zapata, M., García Areiza, O., Sanchez Mora, J., \& Moren Florian, R. (2005). Años de Vida potencialmente perdidos por la población del municipio de Itagui. Año 2005. Medellín.

- Velasquez, A. (2006). Guía metodológica para las estimaciones epidemiológicas del estudio de carga de enfermedad.

- W., H. (1950). A standardized rate for mortality defined in units for lost years of life. Am Journal Publ Health 1950; 40: 17-26.

- WHO. (2011). Closing the gap: policy into practice and social determinants of health.

- Discussion paper ( internet).

- www.aecc.es/sobreelcancer/cancerporlocalizacion/ cancermama/paginas/menopausiaycancerdemama. aspx. (s.f.).

- w w w . a e c c . e s / S O B R E E L C A N C E R/ CANCERPORLOCALIZACION/CANCERMAMA/ PREVENCION/ w w w . a e c c . e s / S O B R E E L C A N C E R /
CANCERPORLOCALIZACION/CANCERMAMA/
PREVENCION/Paginas/factoresderiesgo.aspx. (s.f.). 